\title{
Achieving increased resolution and reconstructed image quality with gradient variance modified super- resolution radial fluctuations
}

\section{Xingyu Gong}

Fudan University

Li Zhou

Fudan University

Longfang Yao

Fudan University

\section{Li Zhang}

Fudan University

\section{Liwen Chen}

Fudan University

\section{Yiyan Fei}

Fudan University https://orcid.org/0000-0002-2413-130X

\section{Lan Mi}

Fudan University https://orcid.org/0000-0002-2320-2225

\section{Baoju Wang}

South China Normal University

Jiong Ma ( $\nabla$ jiongma@fudan.edu.cn )

Fudan University

\section{Article}

Keywords: Degree of Gradient Convergence, Temporal Fluctuation Analysis, Localization Microscopy Methods, Artifacts and Background Noise

Posted Date: July 2nd, 2021

DOI: https://doi.org/10.21203/rs.3.rs-608461/v1

License: (c) (1) This work is licensed under a Creative Commons Attribution 4.0 International License. Read Full License 
1 Achieving increased resolution and reconstructed image quality with gradient

\section{variance modified super-resolution radial fluctuations}

Xingyu Gong ${ }^{1,5}$, Li Zhou ${ }^{2,5}$, Longfang Yao ${ }^{1}$, Li Zhang ${ }^{2}$, Liwen Chen ${ }^{1}$, Yiyan Fei ${ }^{1}$, Lan Mi ${ }^{1}$ *, Baoju Wang ${ }^{3, *}$ and Jiong Ma ${ }^{1,2,4, *}$

${ }^{1}$ Department of Optical Science and Engineering, Shanghai Engineering Research Center of Ultraprecision Optical Manufacturing, Key Laboratory of Micro and Nano Photonic Structures (Ministry of Education), Green Photoelectron Platform, Fudan University, 220 Handan Road, Shanghai 200433, China.

${ }^{2}$ Shanghai Engineering Research Center of Industrial Microorganisms, The Multiscale Research Institute of Complex Systems (MRICS), School of Life Sciences, Fudan University, 220 Handan Road, Shanghai 200433, China

${ }^{3}$ Centre for Optical and Electromagnetic Research, Guangdong Provincial Key Laboratory of Optical Information Materials and Technology, South China Academy of Advanced Optoelectronics, South China Normal University, Guangzhou 510006, China.

${ }^{4}$ Institute of Biomedical Engineering and Technology, Academy for Engineer and Technology, Fudan University, 220 Handan Road, Shanghai 200433, China

${ }^{5}$ These authors contributed equally: Xingyu Gong and Li Zhou *email: lanmi@fudan.edu.cn; baoju.wang@m.scnu.edu.cn; jiongma@fudan.edu.cn

\section{Abstract}

Based on the calculation of the degree of gradient convergence, the superresolution radial fluctuations (SRRF) algorithm can achieve higher resolution by combining temporal fluctuation analysis with localization microscopy methods. The algorithm is also capable of processing high-density fluorescence images. However, there are considerable artifacts due to high density, which lead to a loss of image resolution and low fidelity of images. This study demonstrates the use of fluorescence gradient fluctuations in super-resolution analysis and proposes gradient variance modified SRRF (gmSRRF) algorithm. The gmSRRF algorithm resolves finer structures and compensates for the loss of resolution caused by artifacts in SRRF images using relatively high-density stochastic optical reconstruction microscopy (STORM) data and conventional widefield, confocal, or structured illumination microscopy (SIM) imaging 
sequences. The effectiveness of this algorithm is proven by means of relevant simulations and experiments, which allow the reconstructed SRRF image to improve resolution and reduce artifacts and background noise.

\section{Introduction}

In the last two decades, optical super-resolution imaging techniques have made rapid and significant progress. The theoretical diffraction limit has been overcome using several well-established techniques. One of these techniques is single-molecule localization microscopy (SMLM), which is based on the detection of a single molecule ${ }^{1}$ and typically includes stochastic optical reconstruction microscopy (STORM) ${ }^{2,3}$ and photoactivated localization microscopy $(\mathrm{PALM})^{4}$, 5. Other approaches include stimulated emission depletion (STED) microscopy 6,7 and structured illumination microscopy (SIM) $)^{8,9}$, known as point spread function (PSF) modulation techniques.

STORM and PALM need a great deal of time for super-resolution imaging, and the samples require complicated preparation. Faster reconstruction algorithms for SIM have been demonstrated ${ }^{10,11}$, and STED can be used for dynamic live-cell imaging ${ }^{12,13}$. However, their resolutions are relatively inferior to those of STORM and PALM imaging. SIM and PALM also require stable and precise microscopy systems and complex setups.

Fluorescence signal processing methods with faster and simplified systems have recently emerged. Typical algorithms include the Bayesian analysis of blinking and bleaching $(3 \mathrm{~B})^{14}$, super-resolution optical fluctuation imaging $(\mathrm{SOFI})^{15}$ and super- 
resolution radial fluctuations $(\mathrm{SRRF})^{16}$. 3B can reach a resolution of $\sim 50 \mathrm{~nm}$, but requires a large amount of calculation. For example, $6 \mathrm{~h}$ were required to analyze a 1.5 $\times 1.5 \mu \mathrm{m}$ region $^{14}$. SOFI can achieve a resolution of $\sim 150 \mathrm{~nm}$, but requires thousands of frames. Although SRRF has achieved approximately $105 \mathrm{~nm}$ for widefield and 80$98 \mathrm{~nm}$ for confocal microscopy data using hundreds of slides or less ${ }^{16}$, high-density fluorescence signals will cause artifacts and decrease the actual resolution of the SRRF images.

Here, we present a gradient variance modified SRRF algorithm (gmSRRF) that addresses artifacts by combining SRRF with the temporal statistics analysis of intensity and gradient fluctuations. The optimization algorithm was verified using stimulated and experimental data. Compared with the SRRF algorithm, the gmSRRF algorithm was proven to achieve better super-resolution reconstructed images with fewer artifacts caused by high density and background noise. Additionally, it was applicable to widefield and confocal data.

\section{Algorithm and simulation sections}

There is a conflict between the density of activated fluorophores per frame and the necessary number of frames needed to reconstruct a complete image. SMLM requires low density data for high precision localization, so many frames are needed. SRRF is a super-resolution algorithm that does not depend on the sparse distribution of excited fluorophores ${ }^{17}$. Thus, it can achieve super-resolution with fewer frames than SMLM. It calculates the degree of local gradient convergence (radiality) of an image sequence 
and combines the radiality stack into one reconstructed image through temporal analysis on a sub-pixel scale ${ }^{18}$. SRRF also interpolates the images by a factor of "magnification" before processing. However, for high-density data in areas where structures are close and complex, two or more fluorophores can overlap so that the calculated radiality stack cannot achieve multi-center of radiality. This results in artifacts or even misinterpretation of biological structures in reconstructed images, as shown at the bottom of Fig. 1a. These artifacts cannot be depressed by increasing the number of frames. Fewer frames are necessary to compose a super-resolution image using SRRF if artifacts produced during the processing of high-density data are reduced. Modifying raw data is a feasible way to erase artifacts in SRRF images. Temporally dynamic pixel values in a sequence of fluorescence images contain two major parameters of labeled structures, intensity and gradient, denoted by $U(\mathbf{r}, t)$ and $\mathbf{G}(\mathbf{r}, t)$, respectively. The SRRF algorithm uses radiality to approach intensity peaks without changing the original physical size of the PSF. Thus, its high resolution is based on separate PSFs, which are invalid in high-density situations. The essential issue is resolve two overlapping fluorophores but not to shrink the PSF of an already resolvable fluorophore. SRRF significantly improves the contrast of the images on the basis of the corresponding resolution of exclusive methods, which is exactly the resolution limit of SRRF. While SOFI has demonstrated intensity fluctuations, cumulants can shrink the size of the $\mathrm{PSF}^{15}$ without depending on the detection and localization of isolated fluorophores, as is the case for STORM ${ }^{19,20}$. The time-varying concept can be extended to a gradient when the three conditions adopted by SOFI are met. Through deduction, 
the time-varying properties of the two major parameters are highly related to the

101

102 location of $\mathbf{r}$ in relation to a real fluorescence emitter. Specifically, if $\mathbf{r}$ is located between two close fluorophores:

1. The corresponding $U(\mathbf{r}, t)$ is the sum of the two individual stochastic fluctuations, which fluctuates less than that of a single molecule because of the averaging effect.

2. The gradient vector exhibits a higher variety of orientation and amplitude than at the center of either fluorophore, due to blinking on both sides.

a

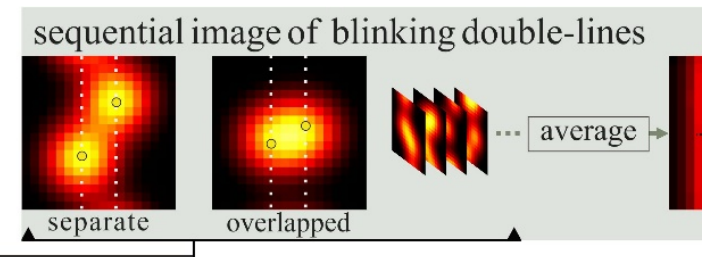

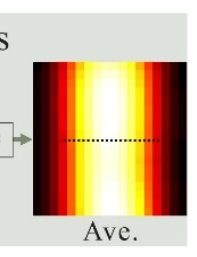
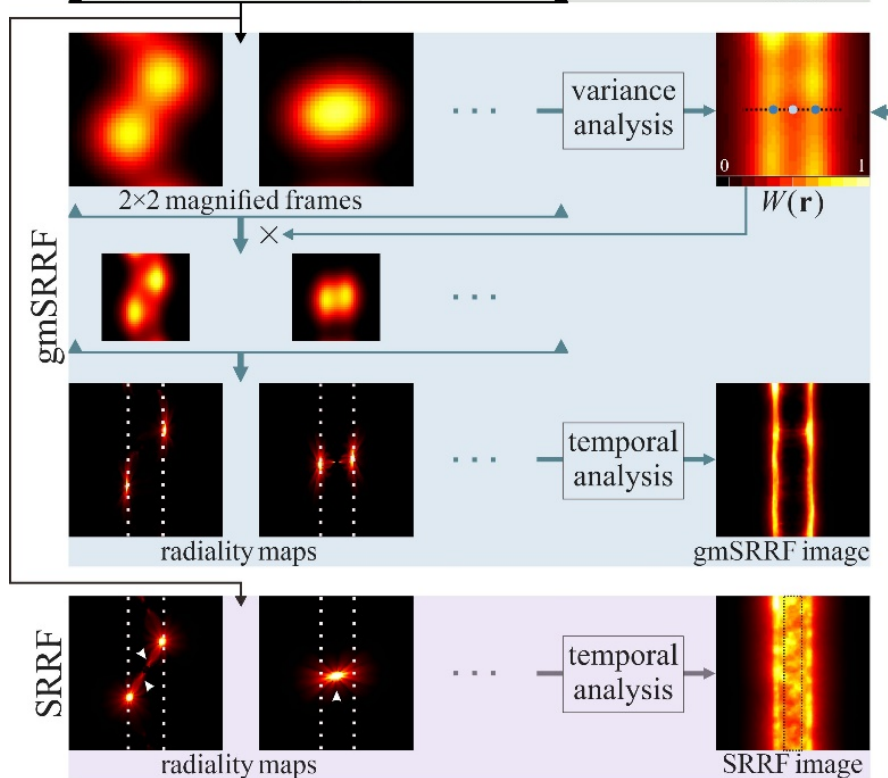

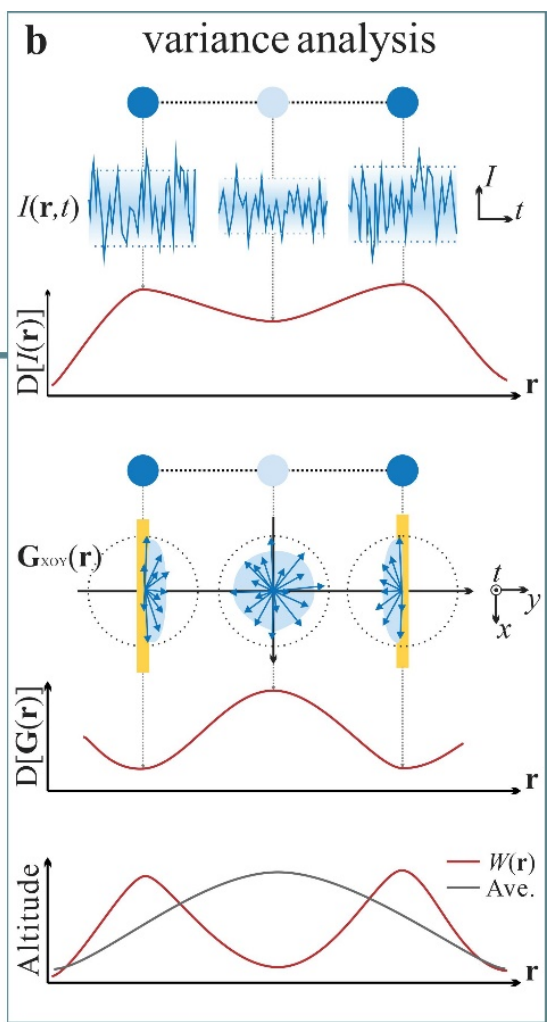

Fig. 1 a Principle of the gmSRRF algorithm and mechanism of artifacts in SRRF when processing a simulated image sequence of a vertical double-line structure labeled with high blinking ratio fluorophores. b Progress of variance analysis using three typical pixel points as examples. The top graph represents the time-varying intensity and its variance curve. The middle graph is a schematic diagram of projections of gradient vectors in every moment (blue arrows) on the XOY plane and its variance curve. Yellow bars indicate the actual position of the double-lines. The bottom graph shows the curves of weighting function and average intensity.

In more general cases, a sample is composed of $N$ single-blinking fluorophores. 
The intensity distribution function is expressed as:

$$
U(\mathbf{r}, t)=\sum_{i}^{N} U\left(\mathbf{r}-\mathbf{r}_{i}\right) a_{i} f_{i}(t)
$$

where $U(\mathbf{r})$ is the point spread function, $a_{i}$ is the maximum brightness of the

fluorophores, and $f_{i}(t)$ is the blinking function, assumed to stochastically range between

0 and 1.

Variance is used as a quantitative indicator to describe the time-varying properties of

$\mathbf{G}(\mathbf{r}, t)$ and $U(\mathbf{r}, t)$. Here, the variance is equivalent to the concept of zero-time lag second-order auto-cumulant ${ }^{21}$. The intensity variance is expressed as:

$$
\begin{aligned}
D[I(\mathbf{r})] & =\left\langle(U(\mathbf{r}, t)-\langle U(\mathbf{r}, t)\rangle)^{2}\right\rangle_{t} \\
& =\left\langle\left(\sum_{i}^{N} U\left(\mathbf{r}-\mathbf{r}_{i}\right) a_{i} \delta f_{i}(t)\right)^{2}\right\rangle_{t} \\
& =\sum_{i}^{N} U\left(\mathbf{r}-\mathbf{r}_{i}\right)^{2} a_{i}{ }^{2}\left\langle\delta f_{i}(t)\right\rangle^{2}{ }_{t} \\
& =\sum_{i}^{N} U\left(\mathbf{r}-\mathbf{r}_{i}\right)^{2} a_{i}{ }^{2} D\left[f_{i}(t)\right],
\end{aligned}
$$

where $\delta f_{i}(t)=f_{i}(t)-\left\langle f_{i}(t)\right\rangle_{t}$ and $D[X]$ denotes the variance of time series $X$.

Fluorophores blink relatively independently, so the time average of the cross-terms is $\left\langle\delta f_{i}(t) \delta f_{j}(t)\right\rangle_{t} \neq 0$ only when $i=j$

Subsequently, we use a Gaussian PSF, and the gradients can be expressed as:

$$
G_{x}(\mathbf{r}, t)=-\frac{\left(x-x_{i}\right)}{\sigma^{2}} U(\mathbf{r}, t)
$$

$$
G_{y}(\mathbf{r}, t)=-\frac{\left(y-y_{i}\right)}{\sigma^{2}} U(\mathbf{r}, t)
$$

Following the same simplification method, gradient variance can then be expressed as:

$$
\begin{aligned}
D[\mathbf{G}(\mathbf{r})] & =D\left[\mathbf{G}_{\mathbf{x}}(\mathbf{r}, t)\right]+D\left[\mathbf{G}_{\mathbf{y}}(\mathbf{r}, t)\right] \\
& =\sum_{i}^{N} \frac{\left|\mathbf{r}-\mathbf{r}_{i}\right|^{2}}{\sigma^{4}} U\left(\mathbf{r}-\mathbf{r}_{i}\right)^{2} a_{i}^{2} D\left[f_{i}(t)\right] .
\end{aligned}
$$

According to the spatial features of the variances of $\mathbf{G}(\mathbf{r}, t)$ and $U(\mathbf{r}, t)$, we define the 
intensity weighting function at position $\mathbf{r}$ as:

$$
W(\mathbf{r})=\frac{D[I(\mathbf{r})]}{D[\mathbf{G}(\mathbf{r})]} .
$$

The intensity weighting function $W(\mathbf{r})$ describes how the gradient variance and intensity variance vary with positions relative to the center of the real PSF. As mentioned above, the numerator reduces and denominator increases if $\mathbf{r}$ is located between two close fluorophores. A low $W(\mathbf{r})$ indicates non-center. When fluorophores fluctuate with the same statistical characteristics, $a_{i} \approx a_{j}, D\left[f_{i}(t)\right] \approx D\left[f_{j}(t)\right]$, and $W(\mathbf{r})$ approximates to a sum of impulse functions. However, this approximation is less precise when the fluorescence signal density increases. The $W(\mathbf{r})$ profile is calculated to improve the resolution of the two overlapping blinking PSFs by a factor of $2 \sqrt{2 \log _{3} 2}(\approx 2.2)$ compared to the PSF profile (Supplementary Text 1$)$. The $W(\mathbf{r})$ profile can better resolve two overlapping PSFs. Therefore, the radiality maps have fewer artifacts if the gradient vector fields of raw image sequence are modified by the $W(\mathbf{r})$ profile. Therefore, we present gmSRRF, which calculates $W(\mathbf{r})$ based on $2 \times 2$ magnified pixels for more precision and fewer interpolation errors, as shown in the middle of Fig. 1a. Subsequently, the reweighted sequence $W(\mathbf{r}) U(\mathbf{r}, t)$ is processed with SRRF instead of the original $U(\mathbf{r}, t)$ sequence. All simulations and experiments follow these same rules:

1. SRRF alone performs 6 times magnification.

2. gmSRRF distributes two times magnification for calculation of the weighting function and three times magnification for SRRF analysis; thus, the total magnification is also 6. 
The Monte Carlo method was used to plot the $W(\mathbf{r})$ and intensity variance $\left(I_{\text {var }}\right)$

160 across two blinking PSFs with different spacings, as shown in Fig. 2a. The full-width

161 half-maximum (FWHM) of the simulated Gaussian PSF was set to $200 \mathrm{~nm}$. The $W(\mathbf{r})$

162 profile showed two resolved peaks when the spacing of the two PSFs was $90 \mathrm{~nm}$, which

163 indicates an approximately 2.2 times improvement in the aspect of spatial resolution.

164 Then, the discrete blinking points were simulated and the image sequence was

165 processed by the SRRF and gmSRRF algorithms. A comparison of the four

166 reconstructed images is shown in Fig. 2b, which indicates that the gmSRRF algorithm

167 has an excellent ability to resolve overlapping molecules. Simulated images of double-

168 lines with different distances $(d)$ were also processed, as seen in Fig. 2d. The gmSRRF

169 images were cleaner with fewer artifacts than the corresponding SRRF images for

170 relatively large spacing $(d=150$ or $120 \mathrm{~nm})$. When the double-lines were close to 90

$171 \mathrm{~nm}$, simple SRRF reconstruction processing caused many artifacts, as shown in Fig. $2 \mathrm{~d}$.

172 At the same time, the profile of the intensity section also revealed an unveracious peak

173 between the double-lines, as seen in Fig. 2e. In contrast, the gmSRRF algorithm reduced

174 artifacts and maintained high fidelity and higher resolution of the reconstructed images. 

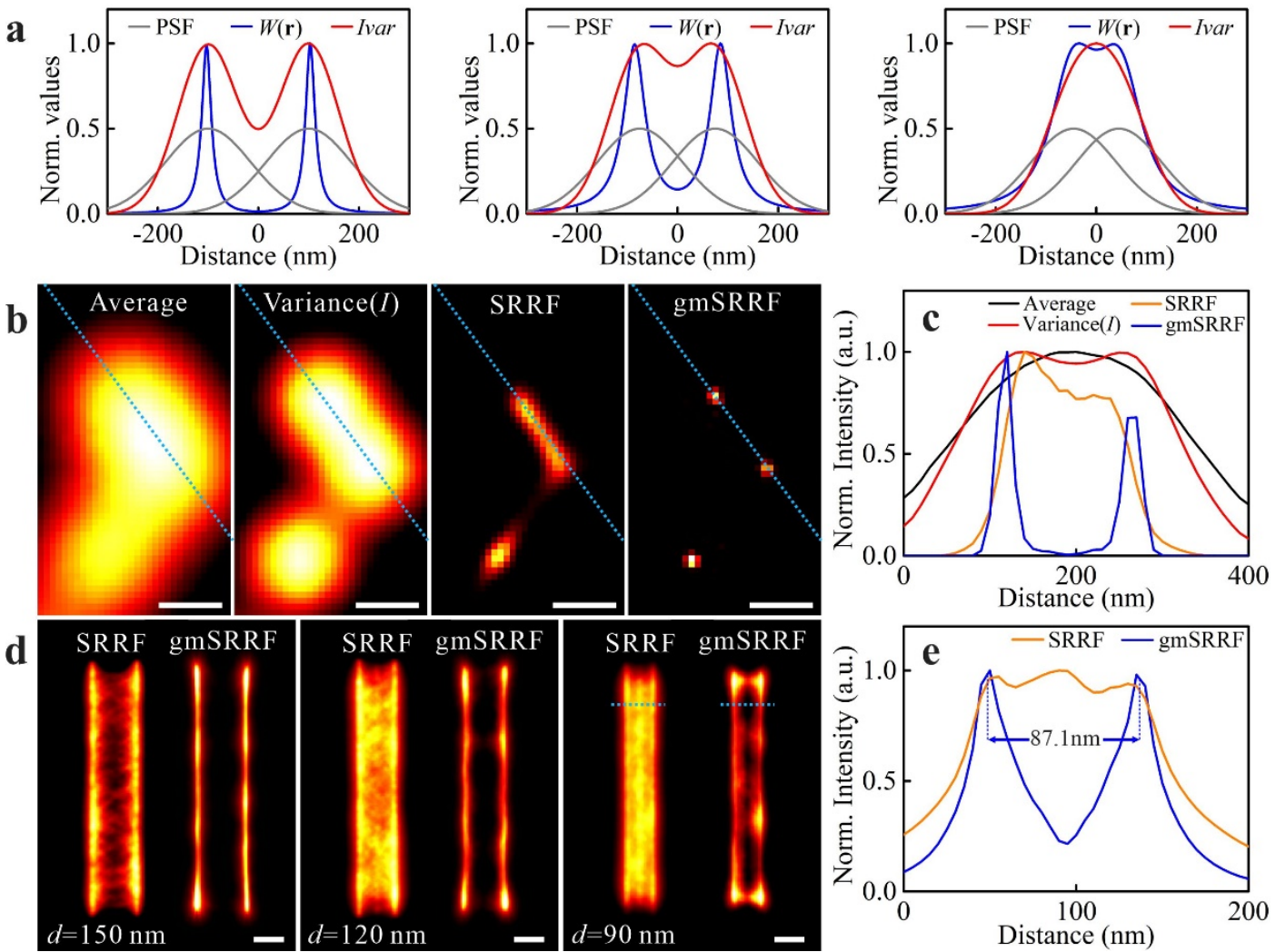

Fig. 2 Simulations of the gmSRRF algorithm. In all simulations, the FWHM of the Gaussian PSF was $200 \mathrm{~nm}$. a From left to right: the $W(\mathrm{r})$ and intensity variance $\left(I_{\text {var }}\right)$ profiles of two simulated blinking PSFs are 200, 150, and $90 \mathrm{~nm}$ apart. Gray lines indicate the positions of the two PSFs. b Comparison of simulated blinking discrete single molecule sequences processed with different methods. From left to right: average intensity, intensity variance, SRRF, and gmSRRF. Scale bars: $100 \mathrm{~nm}$. c The profile of b. d Comparison of double-line image sequences processed with SRRF and gmSRRF. Scale bars: $100 \mathrm{~nm}$. e The profile of resolved double-lines with a $90 \mathrm{~nm}$ space.

\section{Experimental section}

The gmSRRF algorithm was tested on images from high-density STORM data to verify the practical effectiveness of reducing artifacts originating from high density. The algorithm was also applied to widefield, confocal, and SIM data to research the scale of applicability.

First, to verify the effectiveness of the gmSRRF algorithm in terms of eliminating artifacts, high-density STORM raw data were downloaded from the EPFL website ${ }^{22}$. 
SRRF and gmSRRF were applied to the data and the reconstructed images are shown in Figs. $3 \mathrm{~b}$ and c, respectively. The gmSRRF image shows a distinct bimodal profile for two close microtubules, as seen in Fig. 3d, while the SRRF image suffered from bright artifacts and background noise. The labeling density was then set to be extremely high, creating widefield imaging. An Olympus IX83 inverted microscope was used to acquire widefield images of the microtubules, which were labeled with Alexa Fluor 488 by immunofluorescence staining in fixed BSC-1 cells. The gmSRRF image resolved more microtubule structures, which were missing in the SRRF image, as shown in Figs. $3 \mathrm{f}$ and g, respectively. The SRRF algorithm failed to resolve the parallel microtubules shown in the red dashed box because the bright side overpowered the dim side in intensity.
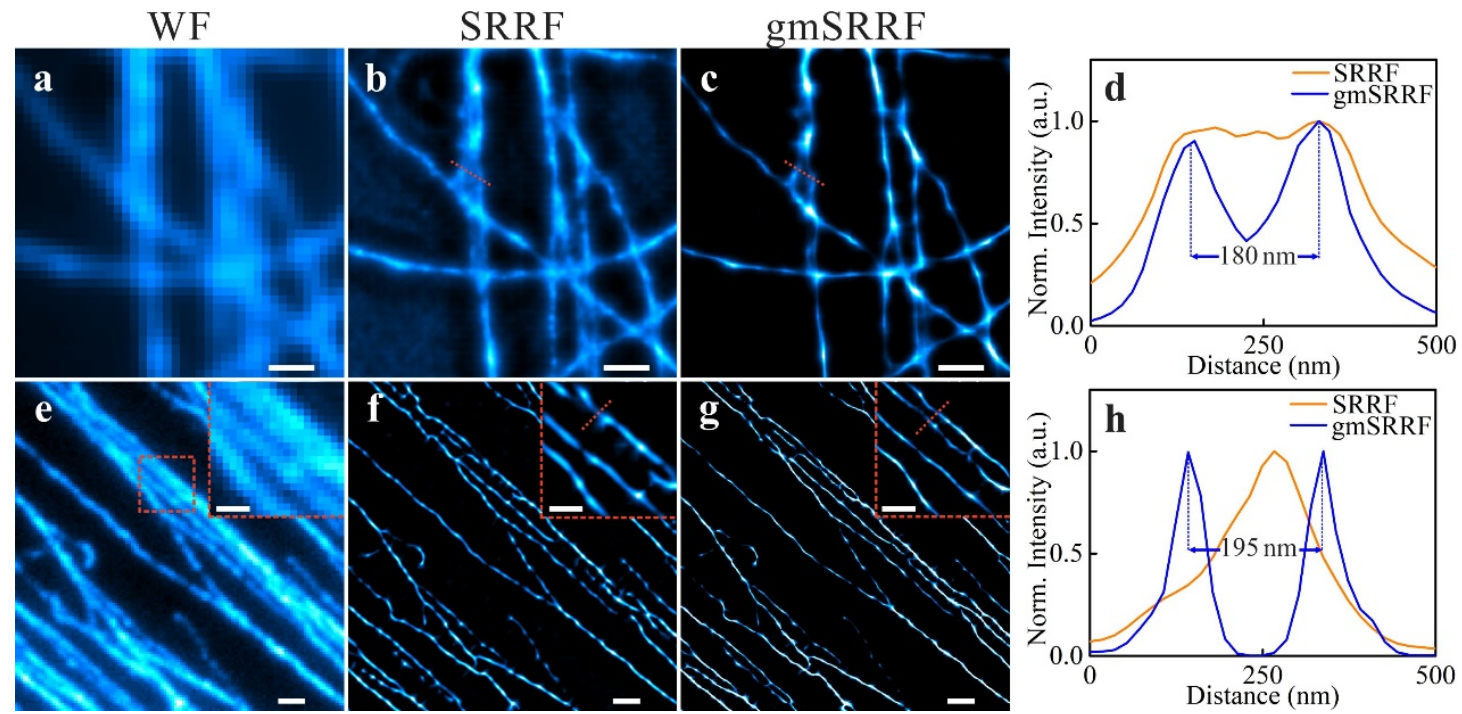

Fig. 3 Comparison of SRRF and gmSRRF under different imaging conditions, such as high-density STORM data and widefield data. a Average image of STORM data served as widefield imaging. $\mathbf{b}$ and $\mathbf{c}$ STORM data was processed with SRRF and gmSRRF algorithms, respectively. d Profiles along the red dotted lines in $\mathbf{b}$ and c. e Widefield image of subcellular microtubules. $\mathbf{f}$ and $\mathbf{g}$ Widefield images processed with the SRRF and gmSRRF algorithms, respectively. $\mathbf{h}$ Profiles along the red dotted lines in $\mathbf{f}$ and $\mathbf{g}$. For a-c: Scale bar is $500 \mathrm{~nm}$. For e-g: Scale bar is $1 \mu \mathrm{m}$ for full view and $500 \mathrm{~nm}$ for region of interest $(\mathrm{ROI})$. 

and improved by the gmSRRF algorithm. Airyscan microscopy from ZEISS can

215 increase the lateral resolution and signal-to-noise ratio (SNR) of the traditional laser 216 scanning confocal microscope ${ }^{23}$. Therefore, we used a ZEISS Airyscan microscope LSM880 for confocal imaging. $\alpha$-tubulin tagged with enhanced green fluorescent protein (EGFP) could denote microtubules as a representative linear structure sample. The confocal image, and reconstructed SRRF and gmSRRF images are shown in Figs. 4a-c, respectively. SRRF produced a Y-shaped misinterpreted structure for the triangular structures, as shown in Fig. 4a. gmSRRF produced a correct profile of the crossing structures, as shown in Fig. 4m. In addition, the lysosome images of Airyscan were acquired. Therefore, the application of the gmSRRF algorithm revealed a number of individual lysosomes that were not well interpreted by the SRRF algorithm, as shown in Figs. 4d-f.

Han et al. applied the SRRF algorithm to 9-frames of raw SIM data and obtained an improved resolution compared with the SIM algorithm ${ }^{24}$. However, 9 frames are inadequate for a high-SNR image. SIM image sequences are accessible for SRRF processing because fast SIM acquisition is already well-established. Therefore, sequential SIM acquisition was adopted in the experimental design. The reduction of

231 SIM artifacts and revelation of hidden structures were proven in microtubule sequential images acquired with SIM, as seen in Figs. 4g-i. The ring-shaped trans-Golgi subcellular structures were also imaged sequentially by SIM for further comparison analysis, and the results are shown in Figs. 4j-1. The phenomenon of loss and 
discontinuity of continuous structures existed in the SRRF image because of uneven

236 fluorescent intensity. In contrast, the gmSRRF image delivered more continuous

237 structures. In addition, SIM images showed considerable pseudo structures around the

238 true cell structures and in the background, as seen in Figs. $4 \mathrm{~g}$ and j, respectively. The

239 artifacts became more severe after processing with the SRRF algorithm. These high-

240 frequency pseudo-patterns caused by raw data intensity issues are commonly seen

241 reconstruction artifacts in $\mathrm{SIM}^{25,26}$. Several algorithms have been proposed to address

242 sidelobe artifacts and improve calculations in the frequency domain ${ }^{27,28}$. gmSRRF

243 appeared to be functional for SIM artifacts in the spatial-temporal domain, filtering out

244 the residual noise signal before it produced artifacts. This is because SIM artifacts

245 exhibit a low stochastic variation in intensity and a relatively high variation in gradient. 

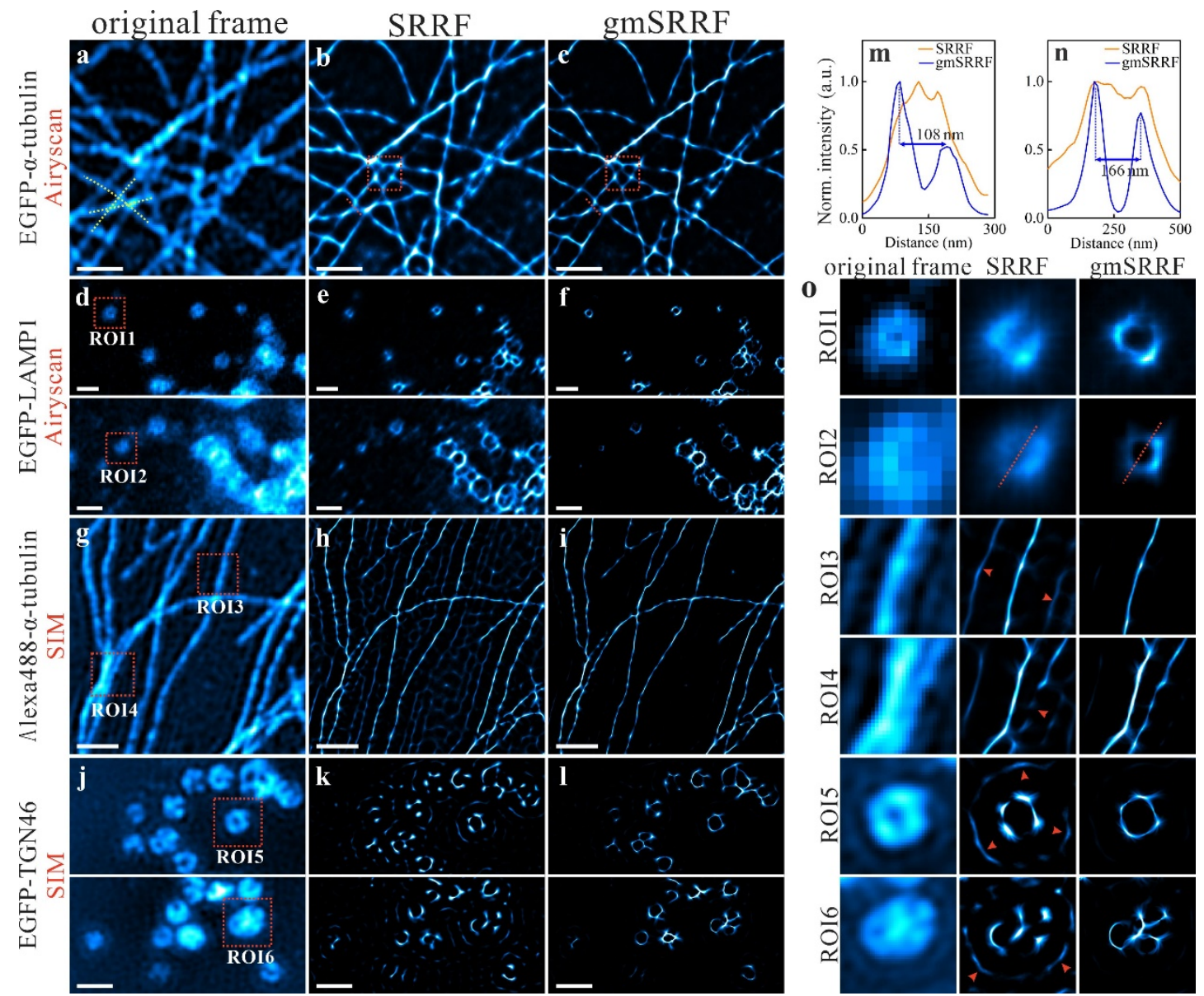

Fig. 4 Resolution and imaging quality improvement in Airyscan images and SIM images. a Airyscan image of microtubules. Yellow dotted lines show a triangular structure formed by three crossing microtubules. b and c Corresponding SRRF and gmSRRF reconstructed images, respectively. $\mathbf{d}$ Airyscan sequence images of lysosomes marked by EGFP-LAMP1. The entire field of view was composed of two different regions. $\mathbf{e}$ and $\mathbf{f}$ Corresponding SRRF and gmSRRF reconstructed images, respectively. g SIM sequence of microtubules marked by Alexa Fluor 488. $\mathbf{h}$ and $\mathbf{i}$ Corresponding SRRF and gmSRRF reconstructed images, respectively. j SIM sequence of trans-Golgi marked by EGFP-TGN46. The entire field of view was composed of two different regions. $\mathbf{k}$ and $\mathbf{l}$ Corresponding SRRF and gmSRRF reconstructed images, respectively. $\mathbf{m}$ The profiles along the red dotted lines in $\mathbf{b}$ and $\mathbf{c}$. $\mathbf{n}$ The profiles along the red dotted lines in the detailed SRRF and gmSRRF images in ROI2. o The magnified details of the original SRRF and gmSRRF frames, labeled with ROI1-6. Scale bars: $1 \mu \mathrm{m}$.

\section{Conclusion}

This study proposed an improved gmSRRF algorithm based on fluorescence

fluctuations and blinking that analyzed the intensity variance and gradient variance of 
raw sequential data to calculate the weighting function used for pre-processing. The proposed gmSRRF algorithm could depress the false radial center accumulated in SRRF processing to deal with artifacts caused by high density. Additionally, twodimensional variance analysis proved to be effective in preventing artifacts caused by uneven florescent intensity and lack of accumulation. At the same time, background stochastic noise was significantly reduced because of the high degree of randomness of the gradient vector. High-density STORM data that was processed using the gmSRRF algorithm had fewer artifacts and less background noise. Widefield and confocal images presented finer and more definite structures after processing. SIM, combined with the gmSRRF algorithm, also produced fewer artifacts and balanced images. Overall, the experimental results verified that the gmSRRF algorithm delivered a comprehensive improvement in terms of artifacts compared to the SRRF algorithm for high-density blinking, widefield, confocal fluctuating, and SIM data.

\section{Methods}

Cell culture and transfection. BS-C- 1 cells obtained from the BeNa Collection in China were cultured in Modified Eagle Medium (MEM) (Gibco), supplemented with 10\% (v/v) FBS (Gibco), 100 units/mL penicillin-streptomycin (Gibco), and 1X MEM non-essential amino acids (Gibco). Nearly $48 \mathrm{~h}$ after transfection with Lipofectamine 2000 (Invitrogen) and Opti-MEM (Life Technologies), cells were suspended and seeded on the poly-1-lysine-coated $0.13-0.16 \mathrm{~mm}$ cover glass (Diamond) on the day before fixation, and incubated at $37{ }^{\circ} \mathrm{C}$ with $5 \% \mathrm{CO}_{2}$. 
288 Cell fixing and staining. Before imaging, cells were washed in phosphate buffered 289 saline (PBS) and fixed in 4\% (w/v) paraformaldehyde (Electron Microscopy Sciences) 290 and $0.2 \%(\mathrm{w} / \mathrm{v})$ glutaraldehyde (Life Science) for $15 \mathrm{~min}$. For microtubule 291 immunolabeling, cells were treated with an extracting buffer (0.1 M PIPES, $1 \mathrm{mM}$ 292 EGTA, $1 \mathrm{mM} \mathrm{MgCl}$, and $0.2 \%$ Triton X-100) for $1 \mathrm{~min}$ before fixation. After fixing, 293 the cells were reduced with $10 \mathrm{mM}$ sodium borohydride (Sigma-Aldrich) for $5 \mathrm{~min}$, 294 and permeabilized with $0.5 \%$ Triton X-100 (Sangon) for an additional 5 min. The cells 295 were washed again with PBS and treated with a blocking buffer containing 5\% (w/v) 296 bovine serum albumin (Jackson ImmunoResearch Laboratories) and 0.5\% Triton X297100 in PBS for $30 \mathrm{~min}$. For microtubule immunolabeling, mouse anti-alpha tubulin 298 (ab74696, Abcam) was used as the primary antibody in 1\% BSA for $1 \mathrm{~h}$, then antimouse Alexa 488 (ab150113, Abcam) was used as a secondary antibody in 1\% BSA for an additional $1 \mathrm{~h}$ after washing 3 times. After staining, the specimens were mounted in ProLong Glass Antifade Mountant (Invitrogen, Thermo Fisher Scientific). All the above-mentioned operations were conducted at room temperature.

Simulations. All simulated data in Fig. 2 were generated using a Gaussian PSF profile with a standard deviation of $200 \mathrm{~nm}$. The maximum brightness of all fluorophores was equal, and the fluctuation function $f_{i}(t)$ was a random sequence ranging between 0 and 1. The intensity distribution was pixelated at a pixel size of $30 \mathrm{~nm}$. Specifically, the profiles shown in Fig. 2a were produced through iteration. A new intensity distribution 
was repeatedly generated and added to the previous sequence to produce a new value of the weighting function until it converged to a steady state. The maximum relative error of five consecutive values of the weighting function was less than $10^{-5}$.

Data analysis. All data images were acquired in or converted to tiff format. The variance analysis programs were written in MATLAB 2020a. The ImageJ plugin 'NanoJ SRRF' (version 1.1Stable0) was used.

Variance Analysis. The first step of gmSRRF is variance analysis. Variance analysis was written using a MATLAB script (Varana_21w17a.m, hereinafter referred to as Varana). The Varana script conducts variance analysis and reweights the raw image stack. Compared with the original weighting function $W(\mathbf{r})$, the Varana script adds some details to prevent the potential issue of convergence, and is then compiled into three MATLAB function files.

Ideally, the weighting function will converge to a minor value or zero in non-central areas. However, the focal plane is mixed with the blurred images of defocused structures due to the limitation of axial resolution. Therefore, the background offset appeared to be uneven. The denser the cell structures, the stronger the background. In these areas, the intensity variance tends to be high, while the gradient variance is the opposite because of the integral fluorescent fluctuations. Consequently, a bright background will cause misinterpretation of the cell structures. In any case, background fluctuation is a smooth variation in a large spatial region. Varana used an intensity 
variance threshold to ensure the convergence of the weighting function. Intensity variance was believed to originate from fluctuations in fluorophores only when it exceeded the threshold. The weighting function degenerated from $\mathrm{D}[I(\mathbf{r})] / \mathrm{D}[\mathrm{G}(\mathbf{r})]$ to $\mathrm{D}[I(\mathbf{r})]$ when the intensity variance decreased below the threshold.

SRRF analysis. The basic settings were as follows: ring radius $=0.5$, magnification $=$ 6 or 3 (gmSRRF), and axes in ring $=6$. Advanced settings were default values except for the temporal analysis, which was the temporal radiality average.

\section{References}

1. Moerner, W. E. \& Kador, L. Optical detection and spectroscopy of single molecules in a solid. Phys. Rev. Lett. 62 (21), 2535-2538 (1989). 10.1103/PhysRevLett.62.2535, Pubmed:10040013

2. Rust, M. J., Bates, M. \& Zhuang, X. Sub-diffraction-limit imaging by stochastic optical reconstruction microscopy (STORM). Nat. Methods 3 (10), 793-795 (2006). 10.1038/nmeth929, Pubmed:16896339

3. Huang, B., Wang, W., Bates, M. \& Zhuang, X. Three-dimensional super-resolution imaging by stochastic optical reconstruction microscopy. Science 319 (5864), 810-813 (2008). 10.1126/science.1153529, Pubmed:18174397

4. Betzig, E. et al. Imaging intracellular fluorescent proteins at nanometer resolution. Science $\mathbf{3 1 3}$ (5793), 1642-1645 (2006). 10.1126/science.1127344, Pubmed:16902090

5. Hess, S. T., Girirajan, T. P. \& Mason, M. D. Ultra-high resolution imaging by fluorescence photoactivation localization microscopy. Biophys. J. 91 (11), 4258-4272 (2006). 10.1529/biophysj.106.091116, Pubmed:16980368

6. Hell, S. W. \& Wichmann, J. Breaking the diffraction resolution limit by stimulated emission: stimulated-emission-depletion fluorescence microscopy. Opt. Lett. 19 (11), 780-782 (1994). 10.1364/ol.19.000780, Pubmed:19844443

7. Klar, T. A., Engel, E. \& Hell, S. W. Breaking Abbe's diffraction resolution limit in fluorescence microscopy with stimulated emission depletion beams of various shapes. Phys. Rev. E Stat. Nonlin. Soft Matter Phys. 64 (6 Pt 2), 066613 (2001). 10.1103/PhysRevE.64.066613, Pubmed:11736302

8. Gustafsson, M. G. Surpassing the lateral resolution limit by a factor of two using structured illumination microscopy. J. Microsc. 198 (2), $82-87$ (2000). 10.1046/j.1365-2818.2000.00710.x, Pubmed: 10810003

9. Lal, A., Shan, C. Y. \& Xi, P. Structured Illumination Microscopy Image Reconstruction Algorithm. Ieee J Sel Top Quant 22 (4), 50-63 (2016). 10.1109/JSTQE.2016.2521542

10. Tu, S. et al. Fast reconstruction algorithm for structured illumination microscopy. Opt. Lett. 45 
11. Huang, X. et al. Fast, long-term, super-resolution imaging with Hessian structured illumination microscopy. Nat. Biotechnol. 36 (5), 451-459 (2018). 10.1038/nbt.4115, Pubmed:29644998

12. Westphal, V. et al. Video-rate far-field optical nanoscopy dissects synaptic vesicle movement.

13. Bergermann, F. et al. 2000-fold parallelized dual-color STED fluorescence nanoscopy. Opt.

14. Cox, S. et al. Bayesian localization microscopy reveals nanoscale podosome dynamics. Nat.

15. Dertinger, T. et al. Fast, background-free, 3D super-resolution optical fluctuation imaging

16. Gustafsson, N. et al. Fast live-cell conventional fluorophore nanoscopy with ImageJ through

17. Culley, S., Tosheva, K. L., Matos Pereira, P. \& Henriques, R. SRRF: Universal live-cell super-

18. Laine, R. F. et al. NanoJ: a high-performance open-source super-resolution microscopy toolbox.

19. Geissbuehler, S., Dellagiacoma, C. \& Lasser, T. Comparison between SOFI and STORM.

20. Ovesný, M. et al. ThunderSTORM: a comprehensive ImageJ plug-in for PALM and STORM

21. Dertinger, T. et al. Achieving increased resolution and more pixels with Superresolution Optical

22. Biomedical Imaging Group. Benchmarking of single-molecule localization microscopy

23. Huff, J. The Airyscan detector from ZEISS: confocal imaging with improved signal-to-noise

24. Han, Y. et al. Ultra-fast, universal super-resolution radial fluctuations (SRRF) algorithm for 400 


\section{Acknowledgements}

This work was financially supported by the Ministry of science and technology of the People's Republic of China (China-Serbia bilateral project SINO-SERBIA2018002), Pioneering Project of Academy for Engineering and Technology, Fudan University (gyy2018-001, gyy2018-002), Shanghai Natural Science Foundation (grant No. 20ZR1405100, 20ZR1403700), Science and Technology Research Program of Shanghai (grant No. 19DZ2282100), Shanghai key discipline construction plan (20202022) (grant No. GWV-10.1-XK01), Shanghai Engineering Technology Research Center of Hair Medicine (19DZ2250500), China Postdoctoral Science Foundation (2021M691089).

\section{Author contributions}

J.M. and B.W. conceived the project. B.W. and J.M. designed the experiments and managed the project, L.Zh. prepared the biological cell specimens, L.Y., L.Zh. and L.Ch. provided the plasmid construct of EGFP-Tubulin; X.G. and L.Zh. performed the imaging experiment, X.G. and B.W. conducted the theoretical simulation and data analysis with contributions from J.M., and L.M., X.G., L.Zh., B.W., and J.M. wrote the manuscript. All authors discussed the results and manuscript.

\section{Additional information}

Supplementary Information accompanies this paper.

\section{Conflicts of interest}

There are no conflicts to declare. 


\section{Supplementary Files}

This is a list of supplementary files associated with this preprint. Click to download.

- Varanasourcecode.zip

- Supplementaryinformation.pdf 